\title{
Vorticity-Strain Rate Dynamics and the Smallest Scales of Turbulence
}

\author{
Dhawal Buaria $\oplus^{1,2, *}$ and Alain Pumir $\oplus^{3,2}$ \\ ${ }^{1}$ Tandon School of Engineering, New York University, New York, New York 11201, USA \\ ${ }^{2}$ Max Planck Institute for Dynamics and Self-Organization, 37077 Göttingen, Germany \\ ${ }^{3}$ Laboratoire de Physique, ENS de Lyon, Université de Lyon 1 and CNRS, 69007 Lyon, France
}

(Received 16 August 2021; revised 19 October 2021; accepted 31 January 2022; published 2 March 2022)

Building upon the intrinsic properties of Navier-Stokes dynamics, namely the prevalence of intense vortical structures and the interrelationship between vorticity and strain rate, we propose a simple framework to quantify the extreme events and the smallest scales of turbulence. We demonstrate that our approach is in excellent agreement with the best available data from direct numerical simulations of isotropic turbulence, with Taylor-scale Reynolds numbers up to 1300 . We additionally highlight a shortcoming of prevailing intermittency models due to their disconnection from the observed correlation between vorticity and strain. Our work accentuates the importance of this correlation as a crucial step in developing an accurate understanding of intermittency in turbulence.

DOI: 10.1103/PhysRevLett.128.094501

A defining property of fluid turbulence is the presence of a wide range of dynamically interacting scales that is bounded from above by the largest scales, which are of the order of flow dimension, and from below by the smallest scales, determined by the diffusive action of molecular viscosity. The largest scales transport the bulk of the flow energy and momentum, whereas the smallest scales are responsible for dissipating the flow energy into heat. The net transfer of energy from large to small scales (onto viscous dissipation) occurs via an energy cascade that renders the averaged energy dissipation rate $\langle\epsilon\rangle$ to become independent of (kinematic) viscosity $\nu$, which is also termed "dissipative anomaly." This phenomenology, first proposed by Kolmogorov (1941) [1,2] (K41 henceforth), identifies the smallest scales in the flow as

$$
\eta_{K}=\left(\nu^{3} /\langle\epsilon\rangle\right)^{1 / 4} ; \quad \tau_{K}=(\nu /\langle\epsilon\rangle)^{1 / 2},
$$

where $\eta_{K}$ and $\tau_{K}$ are the Kolmogorov length and time scales, respectively.

While dissipative anomaly has been widely confirmed [3-6], the overall mean-field description of K41 has been invalidated $[7,8]$. This is because the fluctuations of dissipation rate, and velocity gradients in general, exhibit a high degree of spatial and temporal intermittency, with large non-Gaussian excursions from its mean, that become

Published by the American Physical Society under the terms of the Creative Commons Attribution 4.0 International license. Further distribution of this work must maintain attribution to the author(s) and the published article's title, journal citation, and DOI. Open access publication funded by the Max Planck Society. increasingly stronger with the Reynolds number [9-11]. Such extreme events play a crucial role in numerous physical processes [12-15] and are at the center of turbulence theories and models $[7,8,16]$. Simultaneously, it follows that the smallest scales in the flow, putatively corresponding to the extreme events, would be smaller than the Kolmogorov scales defined by Eq. (1) [10,17-20].

Several phenomenological models have been proposed to describe intermittency, with reasonable success in characterizing the statistics of velocity increments for inertial scales [7,8]. However, an accurate description of the smallest scales has remained elusive for two reasons. The first limitation is the insufficiency of well-resolved data across a wide range of Reynolds numbers, since capturing extreme events requires a very stringent smallscale resolution $[10,21,22]$. The second limitation is that of phenomenological models, which typically appeal to adjustable parameters without a clear connection to the dynamics of the Navier-Stokes equations. For instance, it is well-known that extreme gradients are structurally arranged in tubelike vortices [10,23-25]; however, prevailing intermittency theories neither predict this feature nor take it into account in a precise manner.

In this Letter, overcoming the aforementioned limitations, we propose a simple framework to characterize the smallest scales of turbulence based on vortical flow structures while also directly connecting to the underlying Navier-Stokes dynamics. Our predictions are validated with data from state-of-the-art direct numerical simulations (DNS) of the incompressible Navier-Stokes equations, demonstrating excellent agreement. We additionally show that predictions from prior intermittency models are recovered as a limiting case of our framework, and discuss the root of this discrepancy. 


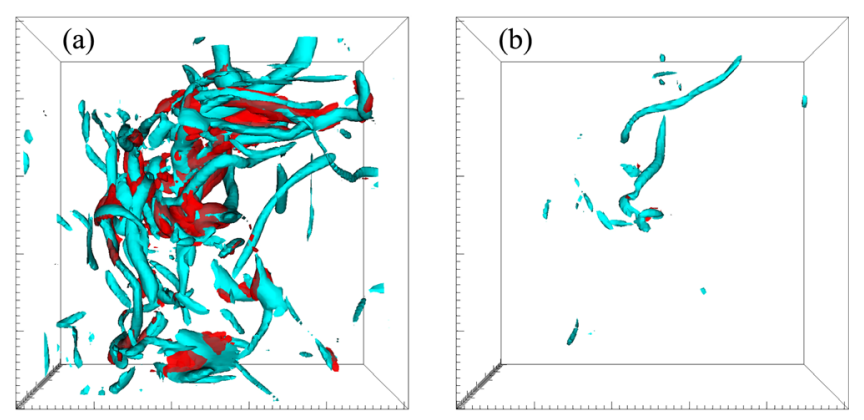

FIG. 1. 3D contour surfaces of $\Omega \tau_{K}^{2}$ (cyan) and $\Sigma \tau_{K}^{2}$ (red) at $R_{\lambda}=1300$. The contour thresholds are (a) 500, and (b) 4000. The domain size in both cases is $\left(100 \eta_{K}\right)^{3}$.

The DNS data used here correspond to the canonical setup of forced stationary isotropic turbulence in a periodic domain [25], enabling the use of highly accurate Fourier pseudospectral methods [26]. The key novelty of our data is that we have simultaneously achieved both very high Reynolds numbers and the prescribed small-scale resolution to accurately resolve the extreme events $[10,22]$. The data correspond to the same Taylor-scale Reynolds number $R_{\lambda}$ range of 140 to 1300 as attained in recent studies [27-31]. However, the run at $R_{\lambda}=1300$ is extended to a grid of $18432^{3}$ points (see [32]) - the largest DNS run to date-presenting a substantial improvement on any previous work investigating the smallest scales $[10,21,33]$. The resolution is $k_{\max } \eta_{K} \approx 6$ for $R_{\lambda} \leq 650$, and $k_{\max } \eta_{K} \approx$ 4.5 for $R_{\lambda}=1300$, where $k_{\max }=\sqrt{2} N / 3$ is the maximum resolved wave number on a $N^{3}$ grid. Convergence with respect to resolution and statistical sampling has been thoroughly established in previous works [10,22,32].

We first identify the intrinsic features of Navier-Stokes dynamics that are essential to characterize the small scales. From the velocity gradient tensor $A_{i j}=\partial u_{i} / \partial x_{j}$, two important descriptors of small-scale motions can be identified: the strain-rate tensor $S_{i j}=\left(A_{i j}+A_{j i}\right) / 2$, and the vorticity vector $\omega_{i}=\epsilon_{i j k} A_{j k} \quad\left(\epsilon_{i j k}\right.$ being the Levi-Civita symbol). We use their square norms

$$
\Sigma=2 S_{i j} S_{i j}, \quad \Omega=\omega_{i} \omega_{i},
$$

the former being the dissipation rate without the viscosity, i.e., $\Sigma=\epsilon / \nu$, and $\Omega$ being the enstrophy. From statistical homogeneity and the definition of $\tau_{K}$, it follows $\langle\Omega\rangle=$ $\langle\Sigma\rangle=1 / \tau_{K}^{2}$. It is well-known that the interaction of strain and vorticity plays a direct role in generating extreme gradients in the flow and hence the smallest scales [24,34]. Understanding the salient properties of this interaction constitutes the first step of our analysis.

Figure 1 shows the structure of extreme events at the highest $R_{\lambda}(=1300)$ via visualization of isosurfaces of strain and vorticity. Figure 1(a) corresponds to a moderately large threshold and illustrates the well-known picture of
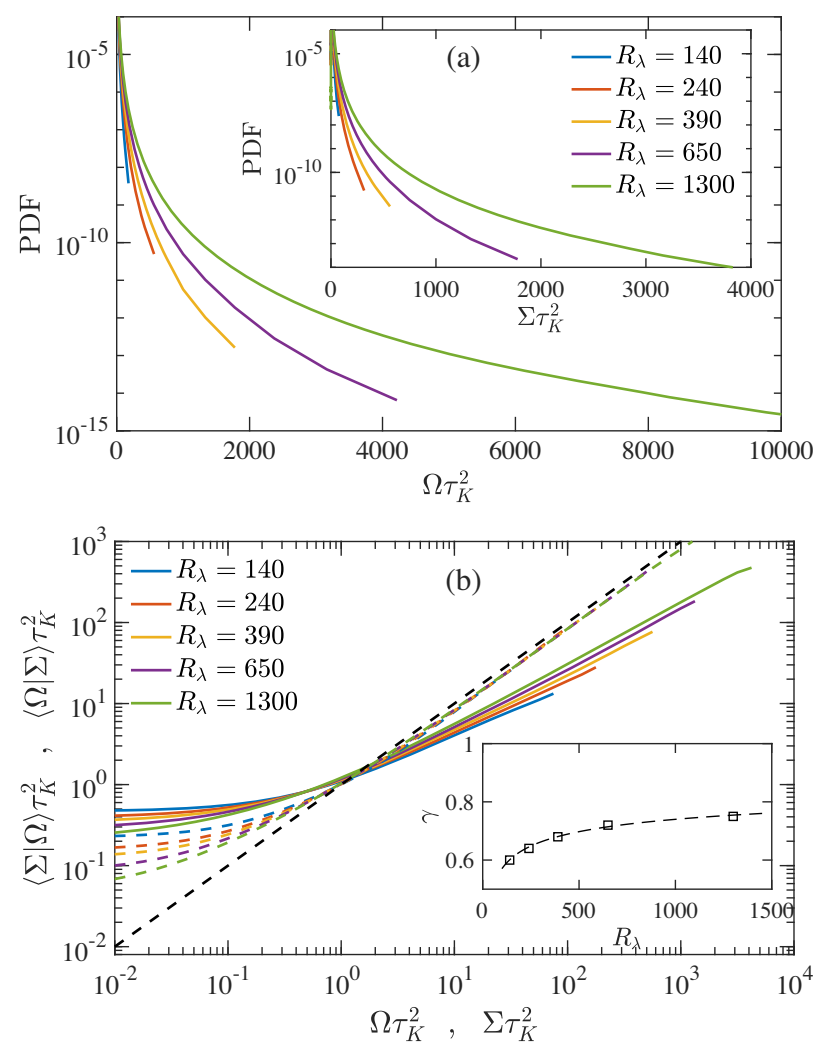

FIG. 2. (a) Probability density functions (PDFs) of $\Omega \tau_{K}^{2}$ and $\Sigma \tau_{K}^{2}$ (inset) for various $R_{\lambda}$. (b) Conditional expectations $\langle\Sigma \mid \Omega\rangle$ (solid lines) and $\langle\Omega \mid \Sigma\rangle$ (dashed lines) for various $R_{\lambda}$. The black dashed line corresponds to a slope of one. Inset shows $\gamma$ as a function of $R_{\lambda}$, for a power law $\langle\Sigma \mid \Omega\rangle \sim \Omega^{\gamma}$ applied in the region $\Omega \tau_{K}^{2} \gtrsim 1$.

intense gradients corresponding to vortical filaments, surrounded by sheetlike regions of intense strain [10,24,25,35]. In Fig. 1(b), a substantially larger threshold is chosen, and remarkably vortical filaments still prevail.

Although $\Omega$ and $\Sigma$ have the same mean, it is well-known that $\Omega$ is more intermittent $[10,25,36]$, likely due to the disparate role of vortex stretching in amplifying vorticity and simultaneously depleting strain [31,34]. This is reflected in their probability density functions (PDFs), shown in Fig. 2(a), which firmly establishes that this difference is not a low- $R_{\lambda}$ effect as previously believed $[21,37,38]$. The local interrelationship between strain and vorticity can be better understood by considering their mutual conditional expectations, shown in Fig. 2(b) (which also have been studied previously in various contexts $[10,21,30])$. The main observation is that while large $\Sigma$ is accompanied by proportionately large $\Omega$, i.e., $\langle\Omega \mid \Sigma\rangle \sim \Sigma$, the converse is not true. Instead, the strain in regions of intense vorticity is considerably weaker, and empirically described by the power law

$$
\langle\Sigma \mid \Omega\rangle \tau_{K}^{2} \sim\left(\Omega \tau_{K}^{2}\right)^{\gamma}, \quad 0<\gamma<1,
$$


where the exponent $\gamma$ slowly increases with $R_{\lambda}$ [see inset of Fig. 2(b)]. Notably, existing intermittency models neither predict this nor take it into account when characterizing the smallest scales.

With the knowledge of vortical flow structures and the asymmetry between the behavior of strain and vorticity (reflected in the exponent $\gamma$ ), we now formulate the framework to quantify the smallest scales in the flow. From a physical standpoint, the smallest length scale in the flow corresponds to the smallest dimension of vortical structures, as obtained from a balance between viscosity and some effective strain $S_{e} \simeq \Sigma_{e}^{1 / 2}$ acting on the particular structure [39]

$$
\eta=\left(\nu^{2} / \Sigma_{e}\right)^{1 / 4}
$$

which can be rewritten as

$$
\eta / \eta_{K}=\left(\Sigma_{e} \tau_{K}^{2}\right)^{-1 / 4}
$$

The classical Kolmogorov result in Eq. (1) is obtained for $\Sigma_{e}$ corresponding to the mean field, i.e., $\Sigma_{e}=\langle\epsilon\rangle / \nu=$ $1 / \tau_{K}^{2}$. Instead, the observation in Fig. 2 suggests using the conditional relation in Eq. (3), leading to

$$
\eta / \eta_{K}=\left(\Omega \tau_{K}^{2}\right)^{-\gamma / 4} .
$$

Introducing the length scale $\eta_{\text {ext }}$ as the size associated with vortex structures corresponding to $\Omega_{\max }$, which in turn corresponds to the smallest timescale $\tau_{\text {ext }}$, i.e., $\Omega_{\max } \sim \tau_{\text {ext }}^{-2}$, leads to

$$
\eta_{\mathrm{ext}} / \eta_{K}=\left(\tau_{\mathrm{ext}} / \tau_{K}\right)^{\gamma / 2} .
$$

Keeping in mind the growth of PDF tails with $R_{\lambda}$ (when normalized by $\tau_{K}$ ), we can write

$$
\eta_{\mathrm{ext}}=\eta_{K} \times R_{\lambda}^{-\alpha}, \quad \tau_{\mathrm{ext}}=\tau_{K} \times R_{\lambda}^{-\beta},
$$

where $\alpha, \beta>0$ are to be determined. Substituting these in Eq. (7) leads to

$$
2 \alpha=\gamma \beta,
$$

giving first direct relation between $\alpha$ and $\beta$.

We now recall that velocity gradients in the flow simply correspond to velocity increments across the smallest length scale. Hence, the strongest gradient simply corresponds to the largest velocity increment, say, $\delta u_{\max }$ over $\eta_{\mathrm{ext}}$ :

$$
1 / \tau_{\mathrm{ext}} \sim \delta u_{\mathrm{max}} / \eta_{\mathrm{ext}}
$$

Based on earlier works $[10,17,24]$ (see also [40]),
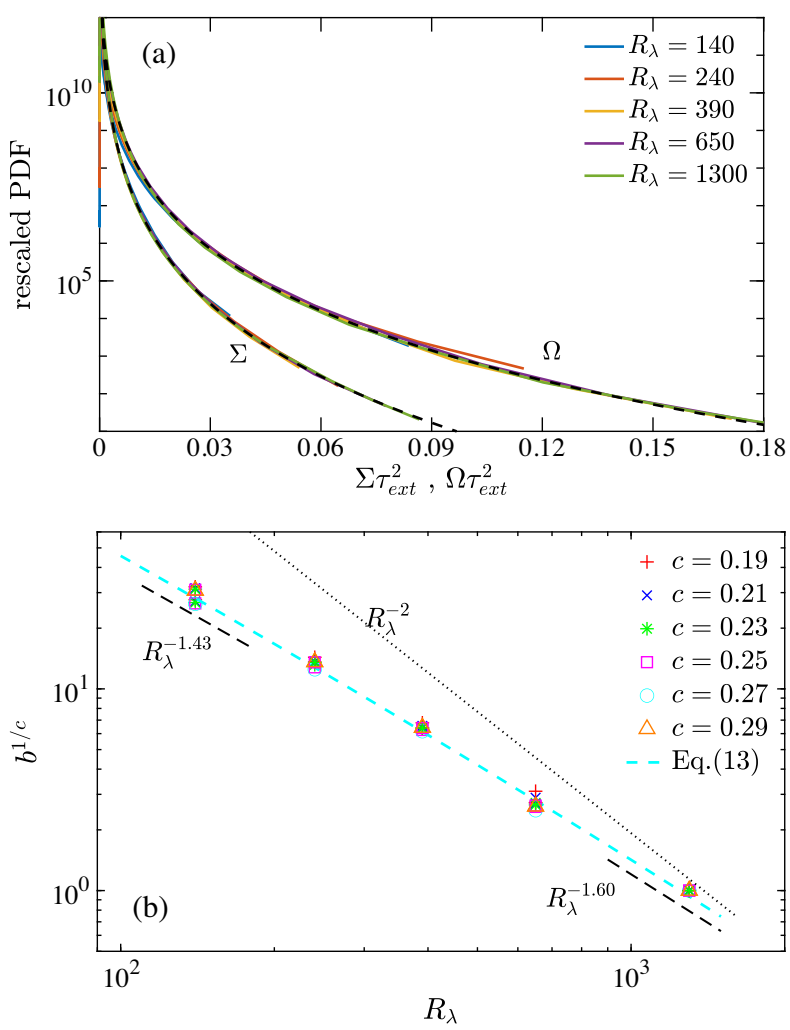

FIG. 3. (a) Rescaled PDFs of $\Omega$ and $\Sigma$, normalized by $\tau_{\text {ext }}^{2}$ corresponding to smallest timescale, at various $R_{\lambda}$. The black dashed line corresponds to stretched exponential fit. (b) Plot of $b^{1 / c}$ vs $R_{\lambda}$ corresponding to stretched exponential fits to PDFs of $\Omega$ and $\Sigma$. For clarity, we have rescaled the curves, so all data points superpose at $R_{\lambda}=1300$. The dashed (cyan) line corresponds to the prediction for $\beta$ in Eq. (13), taking into account the variation of $\gamma$ shown in inset of Fig. 2(b) [40], whereas the dotted line corresponds to the prediction $\beta=1$ in Eq. (19).

$$
\delta u_{\max } \sim u^{\prime},
$$

where $u^{\prime}$ is the rms of velocity, which upon substitution in Eq. (10) gives

$$
\beta=\alpha+1 / 2
$$

Here, we have used the standard estimate $u^{\prime} / u_{K} \sim R_{\lambda}{ }^{1 / 2}$, where $u_{K}=\eta_{K} / \tau_{K}$. Finally, solving Eqs. (9) and (12) allows us to obtain $\alpha$ and $\beta$ in terms of $\gamma$ :

$$
\beta=\frac{1}{2-\gamma}, \quad \alpha=\frac{\gamma}{2(2-\gamma)} .
$$

To first validate the result for $\beta$, we return to the PDFs shown in Fig. 2(a), with the expectation that rescaling them with $\tau_{\text {ext }}$ should collapse the tails. Figure 3(a) shows this result, with $\beta$ (and $\tau_{\text {ext }}$ ) defined based on $\gamma$ obtained in Fig. 2(b), demonstrating excellent agreement. It should be noted that a similar collapse was also obtained in [10] at 
lower $R_{\lambda}$ and for a fixed value of $\beta=0.775$. However, the current data at significantly higher $R_{\lambda}$ negate a fixed value of $\beta$. Hence, the $R_{\lambda}$ dependence of $\beta$ (arising from $\gamma$ ) is a crucial ingredient of the current approach and imperative for obtaining an accurate description. This expectation and the quantitative variation of $\beta$ are also consistent with recent results of [11], which characterize the scaling of extreme dissipation events based on underlying shear-layer structures.

While the arguments leading to Eq. (13) use the physical picture of intense vorticity tubes, the collapse in Fig. 3(a) remarkably indicates that both extreme vorticity and strain scale with $\tau_{\text {ext }}$, though these extrema arise from different spatial locations. This suggests that the amplification of vorticity and strain occurs simultaneously with the same timescale, albeit nonlocally [41], inducing the asymmetry in their local correlation as observed in Fig. 2(b). Thus, the exponent $\gamma<1$, which captures this asymmetry, also captures the nonlocality of vorticity-strain interaction. In fact, as demonstrated later, this is also the key reason for our framework's success over prior intermittency models.

The collapse in Fig. 3(a) can be additionally verified by noting that the tails of PDFs of $\Sigma$ and $\Omega$ (and velocity gradients in general) are well fitted by stretched-exponential functions $[9,10,21,42-44]$ :

$$
f_{X}(x) \approx a \exp \left(-b x^{c}\right),
$$

where $x=\Omega \tau_{K}^{2}$ or $\Sigma \tau_{K}^{2}$. Applying a change of variable $x_{e}=x\left(\tau_{K} / \tau_{\mathrm{ext}}\right)^{2}$ will lead to the transformation $b \rightarrow b^{\prime}=$ $b \times R_{\lambda}{ }^{2 \beta c}$. A necessary condition to collapse the tails would imply that $b^{\prime}$ is independent of $R_{\lambda}$, leading to the expectation that $b^{1 / c} \sim R_{\lambda}^{-2 \beta}$ (for any given value of $c$ ).

Figure 3(b) shows the plot of $b^{1 / c}$ as a function of $R_{\lambda}$, for various $c$ values (comprehensive details about the fitting procedure, and the chosen range of $c$ are discussed in the Supplemental Material [40]). We compare the slope of the data points for $b^{1 / c}$ with the result for $\beta$ in Eq. (13) by using the $\gamma$ obtained earlier from Fig. 2(b) (note $\gamma$ varies from 0.60 to 0.75 for $R_{\lambda}=140-1300$ )—once again, demonstrating excellent agreement. It is worth iterating that the collapse obtained in Fig. 3(a) does not depend on the curve fitting procedure. Nevertheless, this fitting procedure independently reaffirms the robustness of our result and rules out any ambiguity.

While the result for $\tau_{\text {ext }}$ (and $\beta$ ) was readily verified using the PDF tails, verifying $\eta_{\text {ext }}$ (and $\alpha$ ) presents an inherent difficulty. A simple approach would be to evaluate the PDF of the coarse-grained gradient $\delta u_{r} / r$, where $\delta u_{r}$ is the velocity increment over some scale $r$, and successively make $r$ smaller until the PDF of $\delta u_{r} / r$ collapses to the PDF of velocity gradient for $r \leq \eta_{\mathrm{ext}}$. However, DNS data only provides discrete values of $r$ (in integer multiples of the grid spacing $\Delta x$ ), making it impractical to precisely identify the exact $r / \eta_{\text {ext }}$ without invoking some interpolation or

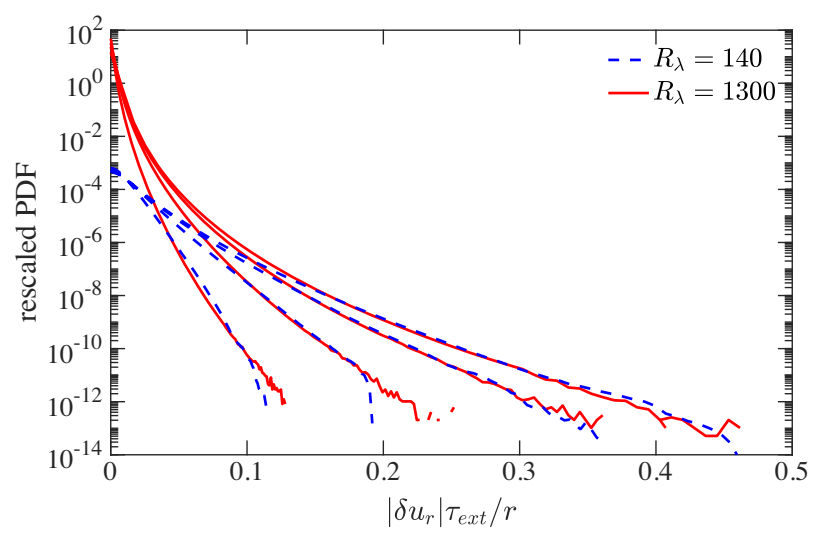

FIG. 4. Rescaled PDFs of the (transverse) velocity increments, $\delta u_{r}$, nondimensionalized by $\tau_{\text {ext }} / r$. Solid red lines are for $R_{\lambda}=1300$, showing $r / \eta_{K}=1,2,4$, and 8 , and dashed blue lines are for $R_{\lambda}=140$, showing $r / \eta_{K}=2,4,8$, and 16 , corresponding to the ratio of $\eta_{K} / \eta_{\text {ext }}$ for these two $R_{\lambda}$. Curves for increasing $r / \eta_{K}$ shift monotonically from right to left. Although not shown, the curves corresponding to the longitudinal increments exhibit similar behavior.

approximate analysis. Instead, we devise a simple alternative by characterizing the deviations of $\delta u_{r} / r$ from the actual gradient. Note, the velocity increment can be longitudinal or transverse, i.e., corresponding to velocity component parallel or perpendicular (respectively) to the separation vector, but it will be evident that this choice is immaterial.

From the Taylor-series expansion of $\delta u_{r}$, it follows that

$$
\frac{\delta u_{r}}{r}=\frac{\partial u}{\partial x}+\frac{\partial^{2} u}{\partial x^{2}} \frac{r}{2 !}+\frac{\partial^{3} u}{\partial x^{3}} \frac{r^{2}}{3 !}+\cdots
$$

For $r \leq \eta_{\mathrm{ext}}$, the rhs converges to $\partial u / \partial x$, whereas for $r>\eta_{\text {ext }}$ deviations are expected due to the higher-order corrections. For the most extreme gradients, we can nominally write $\partial^{n} u / \partial x^{n} \simeq c_{n} u^{\prime} / \eta_{\mathrm{ext}}^{n}$, where $c_{n}$ are independent of $R_{\lambda}$. Together with $u^{\prime} \sim \eta_{\text {ext }} / \tau_{\text {ext }}$, this gives

$$
\frac{\delta u_{r} \tau_{\mathrm{ext}}}{r} \simeq c_{1}+\frac{c_{2}}{2 !}\left(\frac{r}{\eta_{\mathrm{ext}}}\right)+\frac{c_{3}}{3 !}\left(\frac{r}{\eta_{\mathrm{ext}}}\right)^{2}+\cdots,
$$

leading to the expectation that the tails of PDFs of $\delta u_{r} \tau_{\text {ext }} / r$ can be collapsed at different $R_{\lambda}$ by matching the $r / \eta_{\text {ext }}$, thus providing a simpler test with DNS data. We notice that $\eta_{K} / \eta_{\text {ext }}$ at $R_{\lambda}=140$ is approximately 2 times that at $R_{\lambda}=1300$. Figure 4 shows the rescaled PDF of $\delta u_{r} \tau_{\text {ext }} / r$, for $r / \eta_{K}=1,2,4$, and 8 at $R_{\lambda}=1300$, and $r / \eta_{K}=2,4,8$, and 16 at $R_{\lambda}=140$, showing a remarkably good collapse of the tails, providing a strong confirmation of our approach. Similarly, for $R_{\lambda}=140$ and $R_{\lambda}=650$, the ratio of their $\eta_{K} / \eta_{\text {ext }}$ is approximately 1.5 , and a similar collapse is obtained (see [40]). 
The framework developed in this work differs from previous phenomenological models, which ignore important features of the Navier-Stokes dynamics. In this regard, the commonly used notion is that the viscous cutoff scale is defined by the phenomenological criteria of local Reynolds number being unity $[7,17,19,20,33]$ :

$$
\delta u_{r} r / \nu \simeq 1 \text {. }
$$

This is essentially an ad hoc extension of the K41 phenomenology, since $u_{K} \eta_{K} / \nu=1$. The velocity increment $\delta u_{r}$ is assumed to be Hölder continuous, akin to multifractality [7]:

$$
\delta u_{r} / u^{\prime} \sim(r / L)^{h},
$$

where $L$ is the large-eddy length and $h$ is the local Hölder exponent. It trivially follows that the smallest scales correspond to the minimum Hölder exponent $h_{\min }$. Since $\delta u_{r} \sim u^{\prime}$ for the smallest scales, or equivalently $h_{\min }=0$ $[7,17,19]$, it can be shown that [40]

$$
\beta=2 \alpha, \quad \beta=\alpha+\frac{1}{2},
$$

giving $\beta=1$ and $\alpha=1 / 2$, in line with previous predictions [17-20].

It can be readily seen that the result in Eq. (19) differs from our results in Eqs. (9) and (12) only by the factor $\gamma$, both being the same if $\gamma=1$, i.e., when strain and vorticity are locally commensurate. While the numerical results clearly demonstrate that $\gamma<1$, the weak increase in $\gamma$ with $R_{\lambda}$ [inset of Fig. 2(b)] is suggestive of a slow approach to $\gamma=1$ when $R_{\lambda} \rightarrow \infty$. However, a nominal extrapolation of the data in Fig. 2(b) suggests that this limit will be reached at extremely large $R_{\lambda}$, beyond what can be achieved experimentally or numerically [40]. In fact, this is in line with previous and recent results that independently reaffirm the shortcomings of the multifractal model $[11,24,27]$.

Since the result in Eq. (18) (for $h=0$, giving $\delta u_{r} \sim u^{\prime}$ ) is consistent across all descriptions, the noted discrepancy arises from the criterion in Eq. (17). For vortex tubes, the smallest scale as set by Eq. (4) is qualitatively similar to the criteria in Eq. (17). However, it does not provide any constraint on the circulation of the vortex, $\Gamma$, implying that the local Reynolds number defined as $R_{\Gamma}=\Gamma / \nu$ is not necessarily unity. Instead, our results indicate $R_{\Gamma} \simeq R_{\lambda}^{1-\beta}$, in qualitative agreement with the results of [24]. Note, for $R_{\lambda} \rightarrow \infty$, the expectation $\gamma, \beta \rightarrow 1$ implies that $R_{\Gamma} \rightarrow$ constant. Thus, the crucial misstep in prevailing intermittency models appears to be its inability to distinguish longitudinal and transverse components and use their local correlation. In fact, previous and recent results have shown that this shortcoming also extends to inertial range, where longitudinal and transverse structure functions exhibit different scaling exponents, contrary to the prediction from the multifractal model [45-48].
In conclusion, we have developed a simple framework to characterize the smallest scales of turbulence that uses the underlying asymmetry between strain and vorticity dynamics of Navier-Stokes equations. We have demonstrated excellent agreement of DNS data with predictions, and shown that our parameterization reduces to predictions from existing intermittency models when the symmetry between strain and vorticity is restored, albeit at extremely large $R_{\lambda}$, which are unattainable on Earth. In this regard, understanding the asymmetry between vorticity and strain appears to be a crucial component to understanding intermittency in turbulence for all practical situations of interest, suggestive of a new avenue of investigation. It would also be pertinent to extend the current framework to turbulent mixing of scalars, where recent results have suggested that the smallest scales in the scalar field also deviate from classical predictions $[49,50]$.

We gratefully acknowledge the Gauss Centre for Supercomputing e.V. for providing computing time on the supercomputers JUQUEEN and JUWELS at Jülich Supercomputing Centre (JSC), where the simulations reported in this Letter were primarily performed. We thank P. K. Yeung and Kiran Ravikumar for generously providing the data at $18432^{3}$, which was generated on Summit under the DOE INCITE 2019 award. A. P. was supported by the TILT project from ANR (Contract No. ANR-20-CE30-0035).

*Corresponding author. dhawal.buaria@nyu.edu

[1] A. N. Kolmogorov, Local structure of turbulence in an incompressible fluid for very large Reynolds numbers, Dokl. Akad. Nauk SSSR 30, 299 (1941).

[2] A. N. Kolmogorov, Dissipation of energy in locally isotropic turbulence, Dokl. Akad. Nauk SSSR 434, 16 (1941).

[3] K. R. Sreenivasan, On the scaling of the turbulence energy dissipation rate, Phys. Fluids 27, 1048 (1984).

[4] K. R. Sreenivasan, An update on the energy dissipation rate in isotropic turbulence, Phys. Fluids 10, 528 (1998).

[5] B. Pearson, P- $\AA$ Krogstad, and W. Van De Water, Measurements of the turbulent energy dissipation rate, Phys. Fluids 14, 1288 (2002).

[6] Y. Kaneda, T. Ishihara, M. Yokokawa, K. Itakura, and A. Uno, Energy dissipation rate and energy spectrum in high resolution direct numerical simulations of turbulence in a periodic box, Phys. Fluids 15, L21 (2003).

[7] U. Frisch, Turbulence: The Legacy of Kolmogorov (Cambridge University Press, Cambridge, 1995).

[8] K. S. Sreenivasan and R. A. Antonia, The phenomenology of small-scale turbulence, Annu. Rev. Fluid Mech. 29, 435 (1997)

[9] C. Meneveau and K. R. Sreenivasan, The multifractal nature of turbulent energy dissipation, J. Fluid Mech. 224, 429 (1991).

[10] D. Buaria, A. Pumir, E. Bodenschatz, and P. K. Yeung, Extreme velocity gradients in turbulent flows, New J. Phys. 21, 043004 (2019). 
[11] G. E. Elsinga, T. Ishihara, and J. C. R. Hunt, Extreme dissipation and intermittency in turbulence at very high Reynolds numbers, Proc. R. Soc. A 476, 20200591 (2020).

[12] G. Falkovich, A. Fouxon, and M. G. Stepanov, Acceleration of rain initiation by cloud turbulence, Nature (London) 419, 151 (2002).

[13] K. R. Sreenivasan, Possible effects of small-scale intermittency in turbulent reacting flows, Flow Turbul. Combust. 72, 115 (2004).

[14] M. Vergassola, E. Villermaux, and B. I. Shraiman, Infotaxis as a strategy for searching without gradients, Nature (London) 445, 406 (2007).

[15] P. E. Hamlington, A. Y. Poludnenko, and E. S. Oran, Intermittency in premixed turbulent reacting flows, Phys. Fluids 24, 075111 (2012).

[16] C. Meneveau, Lagrangian dynamics and models of the velocity gradient tensor in turbulent flows, Annu. Rev. Fluid Mech. 43, 219 (2011).

[17] G. Paladin and A. Vulpiani, Degrees of freedom of turbulence, Phys. Rev. A 35, 1971 (1987).

[18] K. R. Sreenivasan and C. Meneveau, Singularities of the equations of fluid motion, Phys. Rev. A 38, 6287 (1988).

[19] M. Nelkin, Multifractal scaling of velocity derivatives in turbulence, Phys. Rev. A 42, 7226 (1990).

[20] V. Yakhot and K. R. Sreenivasan, Anomalous scaling of structure functions and dynamic constraints on turbulence simulation, J. Stat. Phys. 121, 823 (2005).

[21] D. A. Donzis, P. K. Yeung, and K. R. Sreenivasan, Dissipation and enstrophy in isotropic turbulence: Resolution effects and scaling in direct numerical simulations, Phys. Fluids 20, 045108 (2008).

[22] P. K. Yeung, K. R. Sreenivasan, and S. B. Pope, Effects of finite spatial and temporal resolution in direct numerical simulations of incompressible isotropic turbulence, Phys. Rev. Fluids 3, 064603 (2018).

[23] S. Douady, Y. Couder, and M. E. Brachet, Direct Observation of the Intermittency of Intense Vorticity Filaments in Turbulence, Phys. Rev. Lett. 67, 983 (1991).

[24] J. Jiménez, A. A. Wray, P. G. Saffman, and R. S. Rogallo, The structure of intense vorticity in isotropic turbulence, J. Fluid Mech. 255, 65 (1993).

[25] T. Ishihara, T. Gotoh, and Y. Kaneda, Study of highReynolds number isotropic turbulence by direct numerical simulations, Annu. Rev. Fluid Mech. 41, 165 (2009).

[26] R.S. Rogallo, Numerical experiments in homogeneous turbulence, NASA Technical Memo, 1981.

[27] D. Buaria and K. R. Sreenivasan, Dissipation range of the energy spectrum in high Reynolds number turbulence, Phys. Rev. Fluids 5, 092601(R) (2020).

[28] D. Buaria, E. Bodenschatz, and A. Pumir, Vortex stretching and enstrophy production in high Reynolds number turbulence, Phys. Rev. Fluids 5, 104602 (2020).

[29] D. Buaria, A. Pumir, and E. Bodenschatz, Self-attenuation of extreme events in Navier-Stokes turbulence, Nat. Commun. 11, 5852 (2020).

[30] D. Buaria and A. Pumir, Nonlocal amplification of intense vorticity in turbulent flows, Phys. Rev. Research 3, L042020 (2021).
[31] D. Buaria, A. Pumir, and E. Bodenschatz, Generation of intense dissipation in high Reynolds number turbulence, Phil. Trans. R. Soc. A 380, 20210088 (2022).

[32] P. K. Yeung and K. Ravikumar, Advancing understanding of turbulence through extreme-scale computation: Intermittency and simulations at large problem sizes, Phys. Rev. Fluids 5, 110517 (2020).

[33] J. Schumacher, Sub-Kolmogorov-scale fluctuations in fluid turbulence, Europhys. Lett. 80, 54001 (2007).

[34] A. Tsinober, An Informal Conceptual Introduction to Turbulence (Springer, Berlin, 2009).

[35] F. Moisy and J. Jiménez, Geometry and clustering of intense structures in isotropic turbulence, J. Fluid Mech. 513, 111 (2004).

[36] R. M. Kerr, Higher-order derivative correlations and the alignment of small-scale structures in isotropic numerical turbulence, J. Fluid Mech. 153, 31 (1985).

[37] G. He, S. Chen, R. H. Kraichnan, R. Zhang, and Y. Zhou, Statistics of Dissipation and Enstrophy Induced by Localized Vortices, Phys. Rev. Lett. 81, 4636 (1998).

[38] M. Nelkin, Enstrophy and dissipation must have the same scaling exponent in the high Reynolds number limit of fluid turbulence, Phys. Fluids 11, 2202 (1999).

[39] J. M. Burgers, A mathematical model illustrating the theory of turbulence, Adv. Appl. Mech. 1, 171 (1948).

[40] See Supplemental Material at http://link.aps.org/ supplemental/10.1103/PhysRevLett.128.094501 for additional details.

[41] Note: vorticity and strain are coupled nonlocally via the Biot-Savart relation [29,30].

[42] R. Benzi, L. Biferale, G. Paladin, A. Vulpiani, and M. Vergassola, Multifractality in the Statistics of the Velocity Gradients in Turbulence, Phys. Rev. Lett. 67, 2299 (1991).

[43] P. Kailasnath, K. R. Sreenivasan, and G. Stolovitzky, Probability Density of Velocity Increments in Turbulent Flows, Phys. Rev. Lett. 68, 2766 (1992).

[44] B. W. Zeff, D. D. Lanterman, R. McAllister, R. Roy, E. H. Kostelich, and D. P. Lathrop, Measuring intense rotation and dissipation in turbulent flows, Nature (London) 421, 146 (2003).

[45] B. Dhruva, Y. Tsuji, and K. R. Sreenivasan, Transverse structure functions in high-Reynolds-number turbulence, Phys. Rev. E 56, R4928 (1997).

[46] X. Shen and Z. Warhaft, Longitudinal and transverse structure functions in sheared and unsheared wind-tunnel turbulence, Phys. Fluids 14, 370 (2002).

[47] T. Gotoh, D. Fukayama, and T. Nakano, Velocity field statistics in homogeneous steady turbulence obtained using a high-resolution direct numerical simulation, Phys. Fluids 14, 1065 (2002).

[48] R. Grauer, H. Homann, and J.-F. Pinton, Longitudinal and transverse structure functions in high-Reynolds-number turbulence, New J. Phys. 14, 063016 (2012).

[49] D. Buaria, M. P. Clay, K. R. Sreenivasan, and P. K. Yeung, Small-Scale Isotropy and Ramp-Cliff Structures in Scalar Turbulence, Phys. Rev. Lett. 126, 034504 (2021).

[50] D. Buaria, M. P. Clay, K. R. Sreenivasan, and P. K. Yeung, Turbulence is an Ineffective Mixer when Schmidt Numbers are Large, Phys. Rev. Lett. 126, 074501 (2021). 